\title{
Deep-brain magnetic stimulation promotes adult hippocampal neurogenesis and alleviates stress-related behaviors in mouse models for neuropsychiatric disorders
}

Yan Zhang ${ }^{1}$, Rong-Rong Mao ${ }^{2}$, Zhi-Fang Chen ${ }^{1}$, Meng Tian², Da-Li Tong ${ }^{1}$, Zheng-Run Gao ${ }^{1}$, Min Huang ${ }^{1}$, Xiao Li ${ }^{1}$,

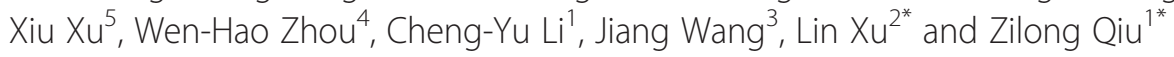

\begin{abstract}
Background: Repetitive Transcranial Magnetic Stimulation (rTMS)/ Deep-brain Magnetic Stimulation (DMS) is an effective therapy for various neuropsychiatric disorders including major depression disorder. The molecular and cellular mechanisms underlying the impacts of rTMS/DMS on the brain are not yet fully understood.

Results: Here we studied the effects of deep-brain magnetic stimulation to brain on the molecular and cellular level. We examined the adult hippocampal neurogenesis and hippocampal synaptic plasticity of rodent under stress conditions with deep-brain magnetic stimulation treatment. We found that DMS promotes adult hippocampal neurogenesis significantly and facilitates the development of adult new-born neurons. Remarkably, DMS exerts anti-depression effects in the learned helplessness mouse model and rescues hippocampal long-term plasticity impaired by restraint stress in rats. Moreover, DMS alleviates the stress response in a mouse model for Rett syndrome and prolongs the life span of these animals dramatically.
\end{abstract}

Conclusions: Deep-brain magnetic stimulation greatly facilitates adult hippocampal neurogenesis and maturation, also alleviates depression and stress-related responses in animal models.

Keywords: Deep-brain magnetic stimulation, Adult hippocampal neurogenesis, Long-term potentiation, Depression, $\mathrm{MeCP} 2$, Rett syndrome

\section{Background}

Transcranial magnetic stimulation (TMS) is a noninvasive approach of brain stimulation, which utilizes an insulated coil placed over the scalp and induces neural activity within the brain [1-3]. TMS is commonly applied in single, paired or repetitive trains. Repetitive TMS (rTMS) has been proven to modulate motor skills and cognitive function in healthy subjects and exhibits therapeutic effects for patients with neurological and psychiatric disorders $[4,5]$. Daily prefrontal TMS was approved

\footnotetext{
* Correspondence: zqiu@ion.ac.cn; Ixu@vip.163.com

${ }^{1}$ Institute of Neuroscience, Shanghai Institutes for Biological Sciences, Chinese Academy of Sciences, Shanghai, China

${ }^{2}$ Key Laboratory of Animal Models and Human Disease Mechanisms, Laboratory of Learning and Memory, Kunming Institute of Zoology, Chinese Academy of Sciences, Kunming, Yunnan, China

Full list of author information is available at the end of the article
}

by Food and Drug Administration (FDA) in 2008 for the treatment of patients with major depressive disorder. Recently, deep-brain magnetic stimulation (DMS) with a modified rTMS protocol has been developed and demonstrated to be effective for Parkinson's disease and neuropsychiatric disorders, including depression [6,7]. Despite the indubitable contribution of rTMS/DMS to cognitive and motor functions of nervous system, the molecular and cellular mechanisms underlying the antidepression effects of rTMS/DMS remain largely unknown. The prevailing hypothesis is that rTMS may stimulate neural activity in certain brain regions by modulating the balance between excitatory and inhibitory neurons [8-12].

Depression is a leading cause of psychiatric disability worldwide. During the past decade, the adult neurogenesis hypothesis of depression has been widely accepted, 
which postulated that the decline in adult hippocampal neurogenesis contributes to the pathophysiology of depression, while the biogenesis of adult new-born neurons in the dentate gyrus (DG) of hippocampus is required for the beneficial effects of antidepressant treatment [13-15]. In non-human primates, stress led to a decrease in adult hippocampal neurogenesis, which was rescued by antidepressant treatment [16]. Chronic rTMS treatment exhibited robust anti-depression effects in animal models and might enhance adult neurogenesis under chronic stress $[17,18]$.

Here we show that DMS increases the proliferation of hippocampal neural progenitor cells in the adult brain, promotes the dendritic complexity of new-born neurons and enhances the neuronal activity in hippocampus, indicated by the up-regulation of activity-dependent genes. Moreover, administration of DMS not only alleviates the depression and anxiety-associated behaviors of a mouse model for Rett syndrome, but also strikingly prolongs the lifespan of these animals.

\section{Results and discussion}

DMS promotes adult hippocampal neurogenesis

To examine the effects of DMS on adult hippocampal neurogenesis in wild-type animals, we applied the DMS protocol to 6 weeks old wild-type mice 20 minutes daily. The schematic illustration of the DMS device is shown in (Additional file 1: Figure S1A). Two magnetic stimulating coils are symmetrically placed on the both sides of the mouse cage. The stimulus device can be discharged through two coils to produce a time-varying pulsed magnetic field. The first stimulating procedure (Program1) is shown in Figure 1A. In this strategy, the magnetic flux density is linear gradient. The magnetic pulse is bipolar, as shown in Additional file 1: Figure S1C,E. The second stimulating paradigm (Program 5) is shown in Figure 1B. Different with Program1, the magnetic flux density of Program 5 is uniform alternating linear gradient, both magnetic fields are illustrated in Additional file 1: Figure S1B-E. First, we examined whether DMS stimulation would have global effects on the general health of experimental animals. We measured the body weights of mice receiving either Program 1 (P1) or Program 5 (P5) treatment daily for 3 weeks and found that P1 or P5 treatment didn't affect the body weight changes comparing to naïve mice (Figure 1C).

To investigate the role of DMS in the proliferation of neural stem cells, we applied a short-term bromodeoxyuridine (BrdU) labeling strategy to monitor postnatal hippocampal neurogenesis in the mice subjected to DMS with P1 or P5, respectively. For the thymidine ana$\log$ incorporation assay, BrdU was administered into adult mice 4 times with 2-hour interval by intraperitoneal injection after 4 or 7 days treatment of DMS

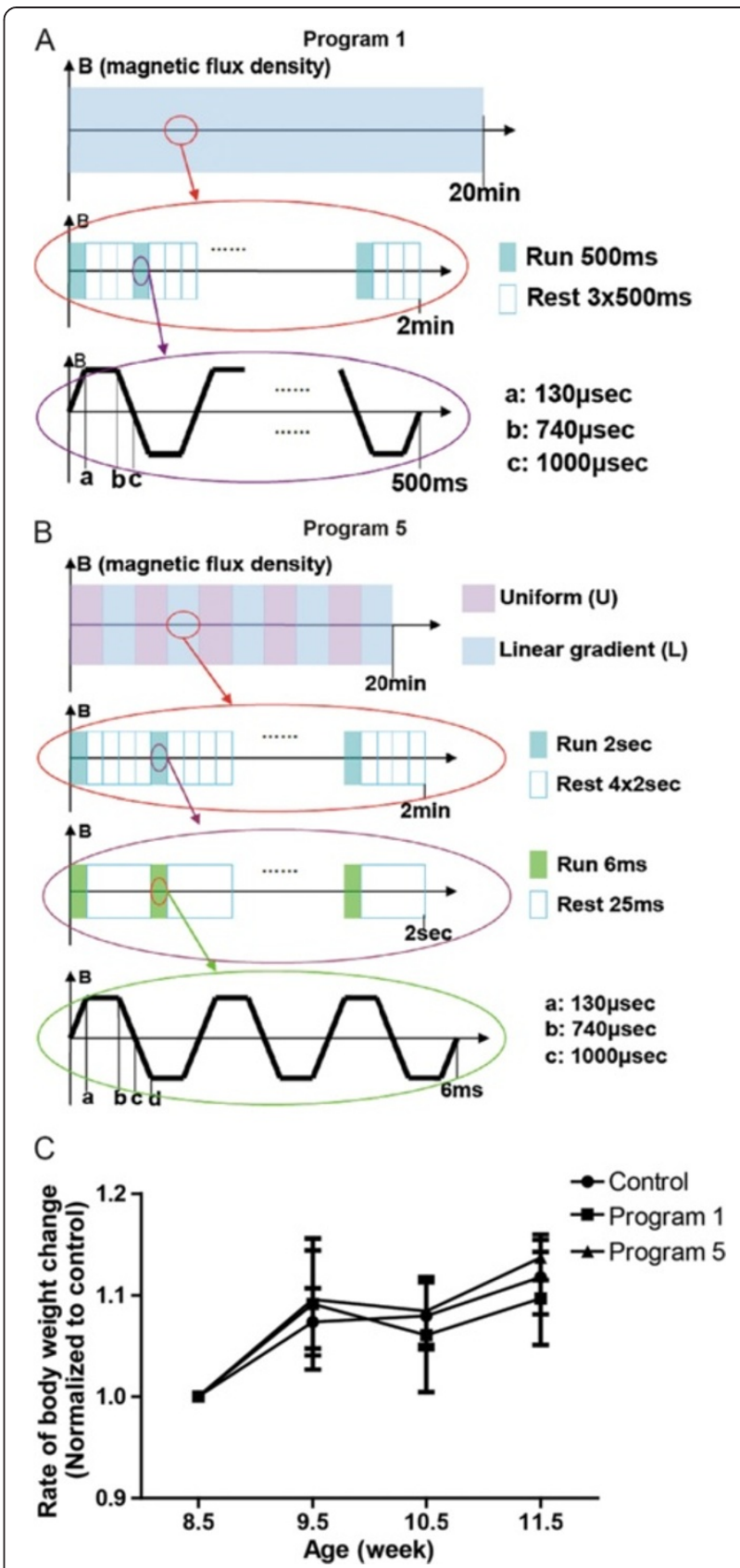

Figure 1 Schematic illustration of the new DMS paradigm. (A) Illustration of Uniform and Linear magnetic fields generated by this equipment. Composition of Program 1 (A) and Program 5 (B). (C) Body weight changes of mice under control, Program1, and Program 5 treatment daily for 3 weeks. ( $n=6$ for each condition).

(Figure 2A). The treated animals were sacrificed 2 hours after the last time of BrdU injection. The staining of BrdU labels the new-born neurons in the hippocampus. The results showed a dramatic increase in the number of active proliferating neural progenitor cells in the subgranular zone of DG after administration of DMS with P1 for 7 days rather than 4 days (Figure 2B,C). Intriguingly, stimulation 

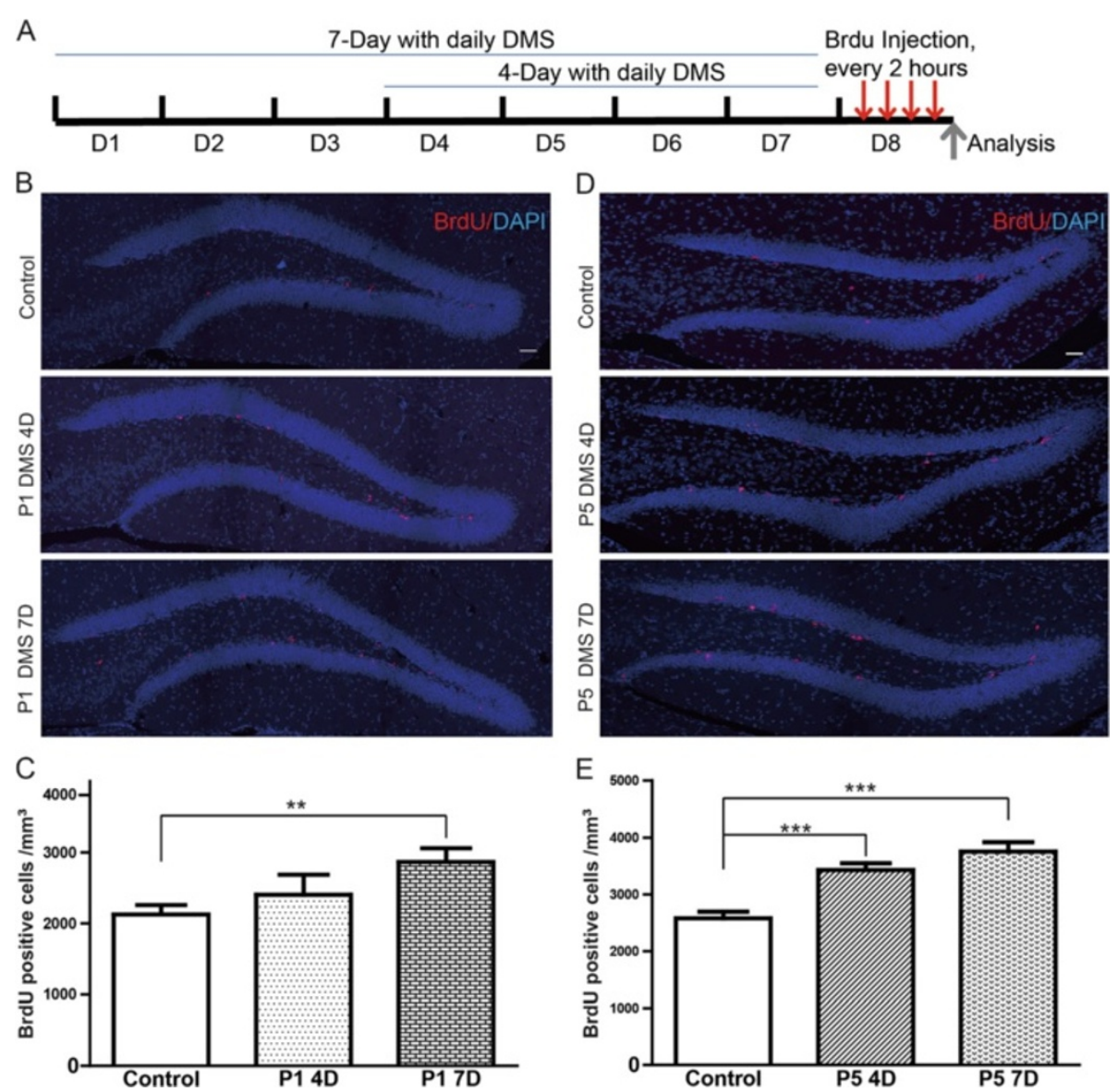

Figure 2 DMS promotes adult hippocampal neurogenesis in rodents. (A) Outline of DMS and BrdU injection experiments. (B) Hippocampal DG BrdU immunostaining on mice with continuous DMS with Program 1 treatment. Red: BrdU; Blue: DAPI staining. (C) Quantification of (B). Values represent mean $( \pm$ ) SEM ( $n=3$ animals for each condition; **: $P<0.005$, two-tailed student's t-test). (D) Hippocampal DG BrdU immunostaining on mice with continuous DMS with Program 5 treatment. Red: BrdU; Blue: DAPI staining. (E) Quantification of (D). Values represent mean ( \pm ) SEM ( $n=3$ animals for each condition; ${ }^{* * *}$ : $P<0.0005$, two-tailed student's t-test). Scale bar $=50 \mu \mathrm{m}$.

with P5 significantly elevated the production of new-born neurons in the DG as early as 4 days after treatment, suggesting that different DMS paradigms may generate various biological effects for adult neurogenesis (Figure 2D,E).

To further confirm whether DMS treatment enhances proliferation of neural progenitors, we further performed immunostaining using antibody against Ki67, a cell proliferating marker on these samples. We found that the number of Ki67-positive cells was significantly increased in DG area after P5 treatment for 7 days, indicating that DMS treatment promotes neural progenitor proliferation (Figure 3A,B). To validate that the BrdU-labeled cells are proliferating progenitors, we performed co-immunostaining for both BrdU and Ki67 and found 100\% of colocalization between the two markers. (Additional file 2: Figure S2A-D). Furthermore, to reveal the cellular identity of BrdU-positive cell we performed immunostaining using antibodies against more neural progenitor markers including radial glial-like cell (RGL) marker glial fibrillary acidic protein (GFAP), immature neuron marker doublecortin (DCX), and intermediate progenitor (IPC) marker T-box brain 2 (Tbr2). We found that cells labeled by short-term BrdU pulse are during actively proliferating process and show 63\% (control) versus 67\% (DMS) co-localization with Tbr2, 21\% (control) versus 16\% (DMS) with GFAP, and 14\% (control) versus 15\% (DMS) with DCX (Additional file 2: Figure S2E-H and Additional file 3: Figure S3A-H). Taken together, our results indicated that DMS promotes not only the self- renewal of RGLs but also their differentiation into IPCs and late neuroblasts.

Next, we examined the effect of DMS treatment on adult hippocampal neurogenesis by in aged animals, in which the rate of adult neurogenesis remains in a much lower level than adult mice. The observation that aging has a negative effect on the proliferation of neural stem cells [19] has called up efforts to boost neurogenesis in senescent animals, which will have beneficial effects for age-related cognitive decline. To study whether DMS 

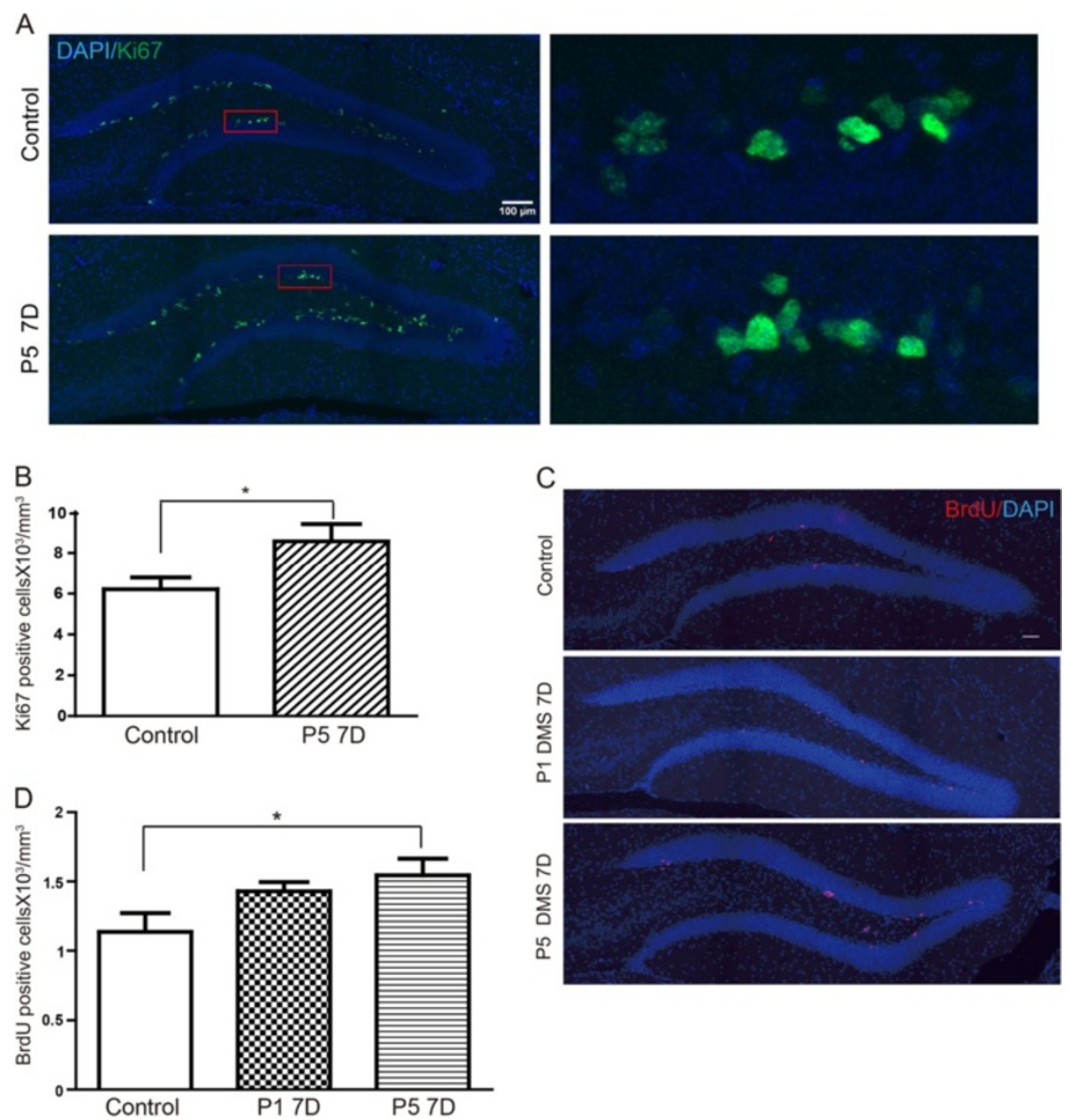

Figure 3 DMS promotes neural progenitors proliferation in rodents. (A) Hippocampal DG Ki67 immunostaining on mice with continuous DMS with Program 5 treatment for 7 days. Blue: DAPl; Green: Ki67 staining. Right panel: Amplification of boxed area of left panel. Scale bar $=100 \mu \mathrm{m}$. (B) Quantification of (A). (C) Hippocampal DG BrdU immunostaining on 9 month old mice with continuous DMS with Program 1 or 5 treatment. (D) Quantification of (C). Values represent mean ( \pm ) SEM (Animal amounts: Control $n=4, P 1 n=3$, P5 n= 4; *: P $<0.05$, two-tailed student's t-test). Scale bar $=50 \mu \mathrm{m}$.

improves the reduced neurogenesis in senescent mice, we applied daily DMS in 9-month old mice for 7 days and examined the neurogenesis by BrdU incorporation assay. Our results revealed that the population of proliferating neural progenitors was significantly enhanced after DMS treatment in P5, while DMS with P1 had a slight increase but not statistically significant effects on hippocampal neurogenesis (Figure 3C,D). Thus, we demonstrated that DMS with patterned stimulation protocols promoted the proliferation of hippocampal neural progenitor cells in both adult and senescent animals.

\section{DMS facilitates the development of new-born neurons}

Adult hippocampal neurogenesis largely recapitulates the process of neural development in embryonic stages. After birth, new-born neurons in the DG migrate into the granule cells layer, extend dendrites toward the molecular layer, project axons through the hilus toward the CA3 and integrate into the existing circuitry $[20,21]$.

To address the effects of DMS on the maturation of new-born neurons, we labeled these neurons by stereotactic injection of retrovirus into the DG of young mice, followed by the 2-week daily treatment of DMS with distinct programs (Figure 4A) [22]. Retroviral infection of proliferating neural progenitor cells with the introduction of green fluorescent protein (GFP) allows the sparse labeling of new-born neurons and the elaborate observation of their morphological complexity. The morphology of GFP labeled new-born neurons was examined with confocal microscopy at 2 weeks post-injection (Figure 4B). The results showed that there was a significant increase in both the number of the tips and total dendritic length after DMS treatment with either P1 or P5, when compared to the control GFP labeled neurons (Figure 4C-E). We 

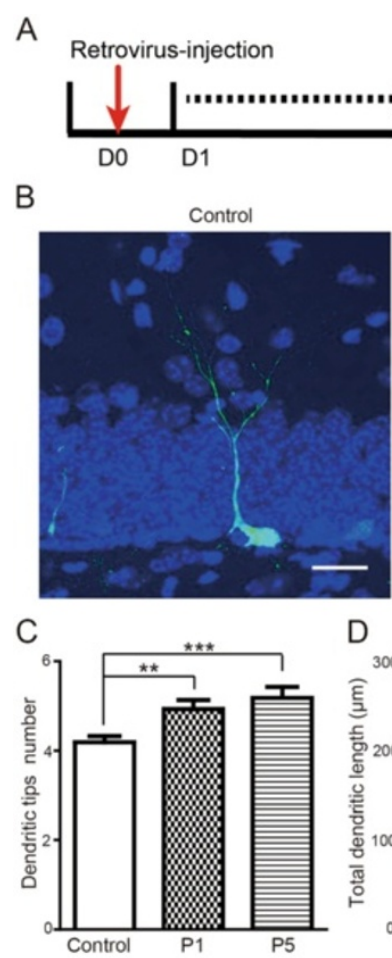

$\mathrm{F}$
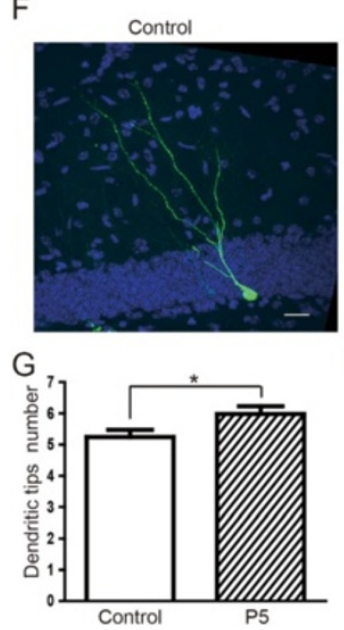

2/4-Week with daily DMS
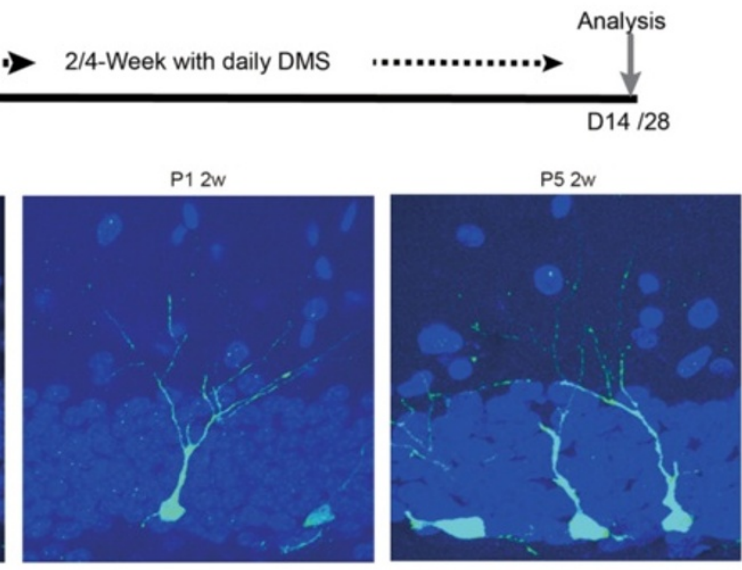

E

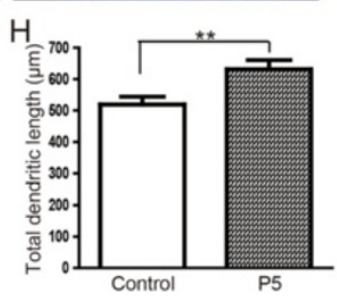

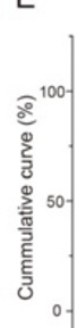

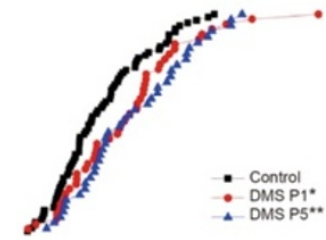

P5 4w
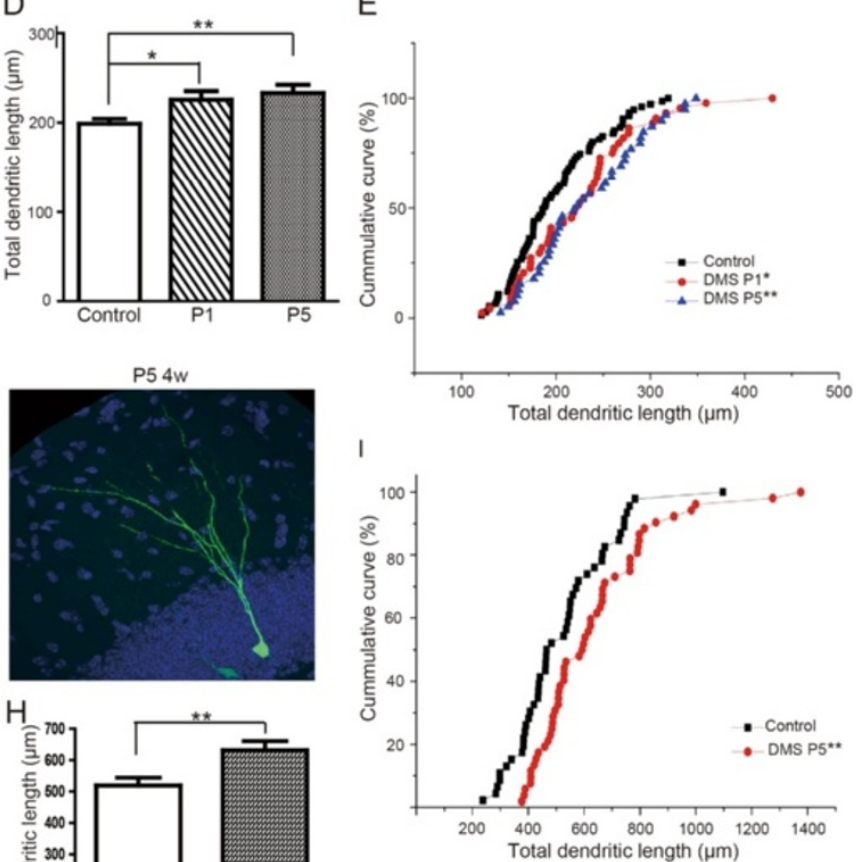

Figure 4 DMS facilitates development of DG new-born neurons. (A) Outline of DMS and retroviral-based new-born neuron label experiments. (B) GFP immunostaining with retroviral labeled new-born neurons after DMS treatment for 2 weeks. Green: GFP; Blue: DAPI staining. (C,D) Total dendritic length and tip numbers measured from (B) Values represent mean $( \pm)$ SEM $\left(n=4\right.$ animals for each group; ${ }^{* *}: P<0.005,{ }^{* * *}$ : $P<0.0005$, two-tailed student's t-test). (E) Accumulative curve of dendritic length of neurons undergoing control, P1 and P5 treatment. (F) GFP immunostaining with retroviral labeled new-born neurons after DMS treatment for 4 weeks. Green: GFP; Blue: DAPI staining. (G,H) Total dendritic length and tip numbers measured from (F) Values represent mean ( \pm ) SEM (Animal amounts, control: $n=4, P 5: n=3 ;{ }^{* *}: P<0.005$, ${ }^{*}: P<0.05$, two-tailed student's t-test). Scale bar $=25 \mu \mathrm{m}$.

further applied DMS with Program 5 up to 4 weeks to mice which received retrovirus injection ahead of stimulation and analyzed the dendritic morphogenesis of new-born neurons (Figure 4F). Consistently, the results revealed that DMS robustly facilitated the dendritic growth and branching of neurons (Figure 4G-I).
In order to examine whether DMS may affect the development of mature neurons, we performed Golgi staining on the brain sections from control and DMStreated animals (Additional file 4: Figure S4A,B). We measured the spine density of DG granule neurons after DMS for 2 weeks and found that DMS has no effects on 
spine density of fully mature neurons (Additional file 4: Figure S4C,D).

Collectively, these evidences demonstrated that DMS treatment not only enhances the proliferation of adult neural progenitor cells in DG, but also facilitates the development and maturation of new-born neurons, suggesting that new-born neurons induced by DMS are able to incorporate into hippocampal neural circuitry and contribute to neuronal plasticity of the central nervous system.

\section{DMS stimulates gene expression in hippocampus in vivo}

To further explore the consequences at the molecular level caused by DMS treatment, we performed immunostaining and quantitative PCR to examine the change of gene expression in the hippocampus in vivo. Neural activity induces the modification of synaptic proteins, promotes local protein synthesis within dendrites and activates gene transcription in neurons. Therefore, we compared the expression level of several activity-dependent genes in the hippocampus between control groups and DMS-treated groups. The immediate early genes including c-fos, egr 1 and arc are commonly used as markers for measuring neuronal activity in the brain. We first examined the expression of immediate early gene $c$-fos, in the DG after daily DMS with P5 paradigm for 4 days by immunohistochemistry. The results of immunostaining showed that the population of c-fospositive neurons was significantly increased in DMStreated animals (Figure 5A,B). Additionally, the Fgf1b gene is a brain-specific expressing gene encoding an important neurogenic niche factor in the adult hippocampus, and is induced by electroconvulsive stimulation [23]. We collected the hippocampal samples from animals with or without DMS treatment, further examined the mRNA levels of $f g f 1 b$ in the DG by quantitative PCR. We found that the expression level of $f g f 1 b$ was remarkably elevated after DMS (Figure 5C). Furthermore, we examined the protein level of Fgf1 in hippocampus with DMS treatment for 4 days. Consistently, we found that DMS treatment for 4 days significantly lead to an increase of protein level of Fgf1 in hippocampus (Figure 5D,E). These results suggest that DMS stimulation increases neural activity in the hippocampus, induces the expression of activity-dependent
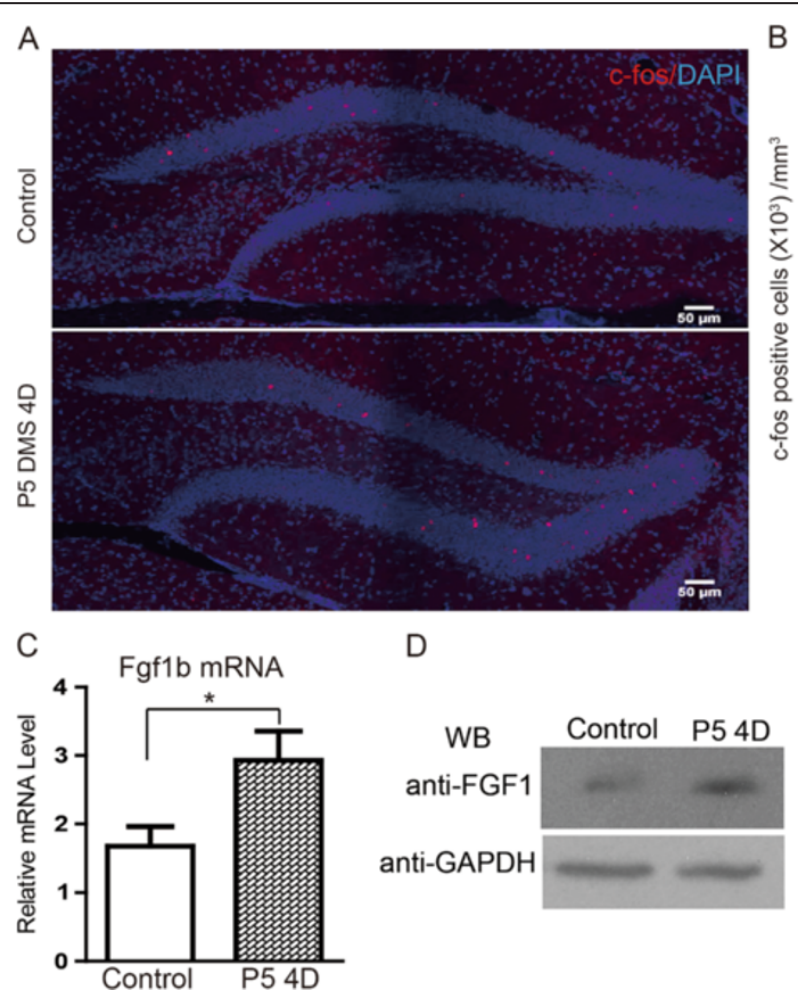

E

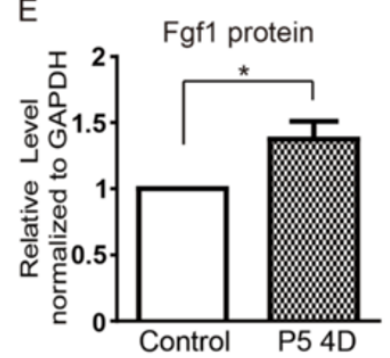

Figure 5 DMS stimulates gene expression in hippocampus. (A) Immunohistochemistry for c-fos expression in hippocampal DG region after DMS with P5 treatment for 4 days. (B) Quantitation for c-fos positive cells in per $1 \mathrm{~mm}^{3}$. Values represent mean $( \pm)$ SEM ( $\mathrm{n}=4$ for each conditions; *: $\mathrm{P}<0.05$, student's t-test). (C) mRNA level of fgf1b gene in hippocampus after DMS with P5 for 4 days. Values represent mean ( \pm ) SEM ( $=4$ for each conditions; *: P<0.05, student's t-test). (D) Protein level of Fgf1b after DMS P5 treatment for 4 days. Hippocampal tissue samples were collected from control and animals received P5 daily treatment for 4 days. Samples were homogenized and analyzed by SDS-PAGE using antibodies indicated. (E) Quantification of (D). 
gene and leads to the release of neurogenic niche factors, which thereby promotes neurogenesis and plasticity in the adult brain.

\section{DMS exerts anti-depression effects on rodent model}

It is postulated that the defects in adult hippocampus neurogenesis is strongly associated with major depressive disorder. Exercise may alleviate depression symptoms via enhancing neurogenesis in animals [24]. The rTMS has been an effective therapeutic tool for the treatment of several neuropsychiatric disorders in human patients, as well as rats with chronic unpredicted mild stress (CUMS) [25]. The evidence that application of DMS to wild type animals increases adult neurogenesis prompted us to investigate whether the newlydeveloped DMS protocol has anti-depressive effects in the rodent model. We applied learned helpless animal paradigm to model some aspects of depression in rodents [26]. Following 2 days of unpredicted electric footshocks, we successfully induce the depression phenotype in mice assessed by forced swimming test, in which mice with foot-shocks appeared to have increased immobility time compared to naïve animals (Figure 6A,B, Additional file 5: Figure S5A-E). After treating mice with continuous 5-day DMS treatment with P5, we found that the immobility time of depressed mice induced by foot-shock was fully rescued to normal level by the DMS treatment (Figure 6B).

The pathophysiology of major depressive disorder has been characterized by alternations of molecular markers. Whole-genome expression profiling revealed that mitogenactivated protein kinase phosphatase-1 (MKP-1) was dysregulated in the hippocampal tissues from patients with depression, suggesting that MKP-1 serves as a molecular marker for depression [27]. Consistently, the expression level of MKP-1 was significantly up-regulated in the hippocampus of mouse model with depression phenotypes (Figure 6C). Importantly, the dys-regulated expression of MKP-1 in depressed mice was completely restored by the DMS treatment (Figure 6C).

Next we investigated whether increased adult hippocampal neurogenesis induced by DMS plays a critical role in alleviation of depressive behavior by elimination of neurogenesis using gamma irradiation (IR). After applying IR after foot shock, we found that IR abolished the effect of DMS on alleviation of depressive behaviors (Figure 6D,E). Meantime, we found that IR itself also leads to a dramatic decrease of hippocampal adult neurogenesis (Additional file 6: Figure S6A-C).

Furthermore, mood disorder induced by acute stress impairs long-term potentiation (LTP) in the neural circuitry of hippocampus [28-31]. The effects on synaptic plasticity occur following a plethora of stressors including administration of shock, exposure to a predator or a novel environment [32]. We used chronic restraint stress paradigm to induce depression in rats and recorded LTP in hippocampus by in vivo electrophysiological recording. Indeed, LTP was found to be impaired in rats undergoing restraint stress (Figure 6F). Notably, DMS treatment for 7 days largely rescued the deficit LTP induction in depressed rats (Figure 6F,G). These behavioral, molecular and electrophysiological evidences strongly support that short-term DMS treatment is effective for alleviating depression-associated phenotypes in animal models.

\section{DMS alleviates anxiety-associated phenotypes in a mouse model for Rett syndrome}

Finally we would like to further examine whether DMS could alleviate the anxiety-related behaviors in other disease models. Rett syndrome is a severe neural developmental disorder, primarily caused by loss-of-function mutations of gene MECP2 (Methyl-CpG-binding protein 2) [33-35]. The mouse carrying a truncated form of MeCP2 protein $\left(m e c p 2^{308 / y}\right)$ mimics severe phenotypes of Rett syndrome patients, including elevated anxiety and stress responses [36]. Thus we asked whether DMS could help to relieve the anxiety-associated phenotypes in mice carrying mecp $2^{308 / y}$ mutation.

We first examine stress responses in meсp $2^{308 / y}$ mouse and its wild type littermates, using light-dark transition paradigm. We found that mice carrying mecp $2^{308 / y} \mathrm{mu}$ tant appeared to spend much less time in light compartment and thus to have increased anxiety levels (Figure 7A,B). Interestingly, chronic daily DMS treatment for 5 months restored elevated anxiety levels of mecp $2^{308 / y}$ mice to normal level (Figure 7A,B). Thus, DMS treatment appears to have significant effects to regulate the anxiety levels of animals for disease models.

Lastly, we asked whether DMS treatment would make positive impacts to the general health of the mouse model of Rett syndrome. Lack of MeCP2 in mouse would lead to significant shortage of lifespan $[37,38]$. We acquired nervous system specific mecp 2 knockout mice by crossing mice carrying neuronal specific Cre transgene nestin-cre with mecp 2 floxed allele- mecp $2^{\text {flox/y }}$. We found that loss of MeCP2 in the nervous system would lead to substantial shortage of lifespan down to around 130 days or so (Figure 7 C). Surprisingly, mice with nestin-cre, mecp $2^{\text {flox/y }}$ received daily DMS with either P1 or P5 appeared to have enormously extended lifespan (Figure 7C), indicating that long-term treatment of DMS indeed exerts positive effects on the general health to mouse model of Rett syndrome.

\section{Discussion}

The growing interest in noninvasive brain stimulation generated by TMS led to its widespread application to treat various neurological and psychiatric disorders 
A

\begin{tabular}{|l|lllllll|}
\hline & Time & D1 & D2 & D3 & D4 & D5 & D6 \\
\hline Treatment & & & & & \\
\hline Doot shock & + & + & - & - & - & - \\
Force swim & $+/-$ & $+/-$ & $+/-$ & $+/-$ & $+/-$ & - \\
\hline
\end{tabular}

B

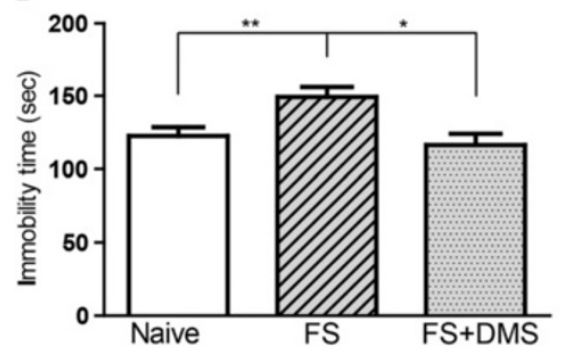

D

\begin{tabular}{|l|lllllll|}
\hline \multicolumn{1}{|c|}{ Time } & D1 & D2 & D3 & D4 & D5 & D6 \\
\hline Treatment & & & & & \\
\hline Foot Shock & + & + & - & - & - & - \\
DMS & $+/-$ & $+/-$ & $+/-$ & $+/-$ & $+/-$ & - \\
Force swim & - & - & - & - & - & + \\
IR & - & + & - & - & - & - \\
\hline
\end{tabular}

$\mathrm{F}$

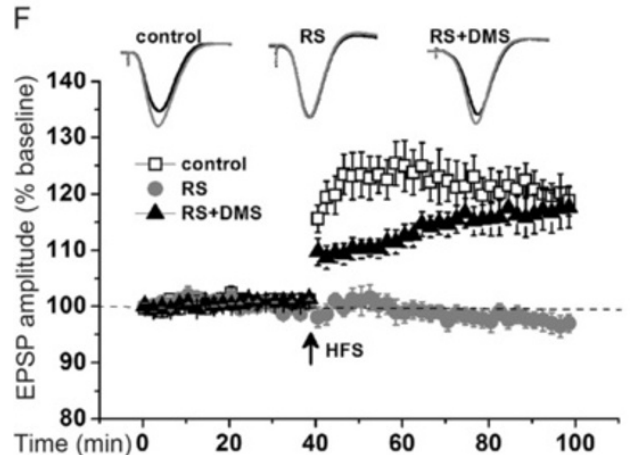

C MKP-1 mRNA
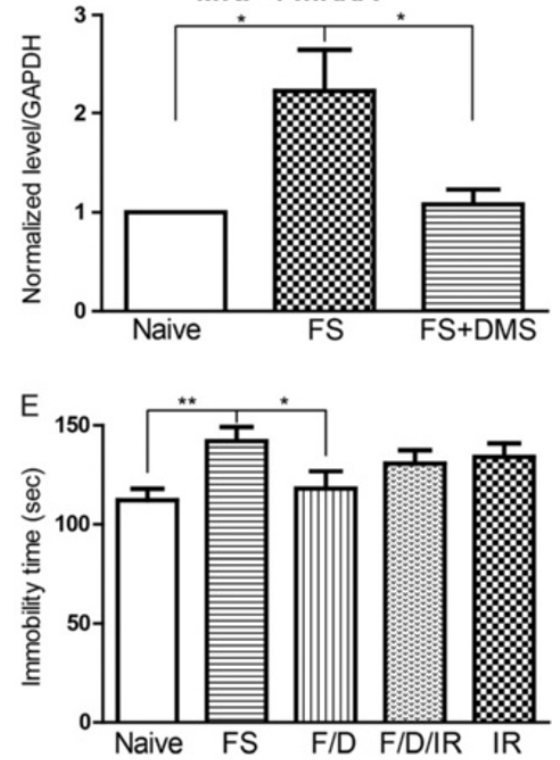

G

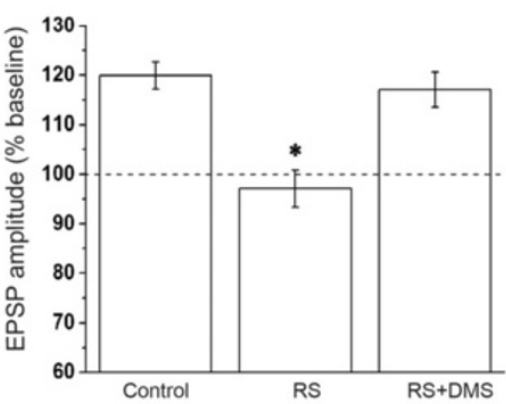

Figure 6 DMS rescues stress-induced phenotypes and synaptic plasticity in rodent models. (A) Outline of foot-shock-induced depressive behavioral paradigm and DMS treatment experiments. (B) Behavioral effects of learned helplessness mouse model with DMS treatment. Values represent mean $( \pm$ ) SEM (Animal amount: control $n=15$, Foot-shock $n=19$, Foot-shock with DMS $n=15$; **: $P<0.005$, two-tailed student's t-test. (C) Hippocampal Mkp-1 mRNA level in a learned helplessness mouse model with DMS treatment. Values represent mean $( \pm)$ SEM $(n=12$ animals for each group; *: P<0.05, two-tailed student's t-test). (D) Outline of foot-shock-induced depressive behavioral paradigm, DMS treatment, and gamma irradiation experiments. (E) Behavioral effects of learned helplessness mouse model with DMS and IR treatment. Values represent mean $( \pm)$ SEM $(n=12-16$ for each condition; **: $\mathrm{P}<0.005$, *: $\mathrm{P}<0.05$, two-tailed student's t-test). (F) DMS treatment restored hippocampal LTP impaired by the chronic restraint stress. Compared with control group ( $n=5,119.92 \pm 2.78 \%$ of baseline ), the LTP induction was significantly impaired in Restraint Stress (RS) group ( $n=5$, $97.10 \pm 1.67 \%$ of baseline) and restored in RS + DMS group ( $n=12,117.06 \pm 3.54 \%$ of baseline); (G) Summary of LTP induction in control, RS and RS + DMS group $\left(F(2,19)=8.64,{ }^{*} p=0.002\right.$, compared with control group). Statistical comparisons in electrophysiological studies were made by the least significance difference test of one-way ANOVA. The significance level was set as *: $p<0.05$.

including major depression and Parkinson's disease. The NeuroStar TMS Therapy system (Neuronetics, Inc., Malvern, PA, USA) received FDA clearance for the treatment of adult patients with intractable depression in 2008. It is assumed that magnetic stimulation makes use of electromagnetic principles to alter neural activity non-invasively, and induces focal as well as network effects in the brain. However, how rTMS changes the cellular behavior and functional connectivity remains enigmatic. 

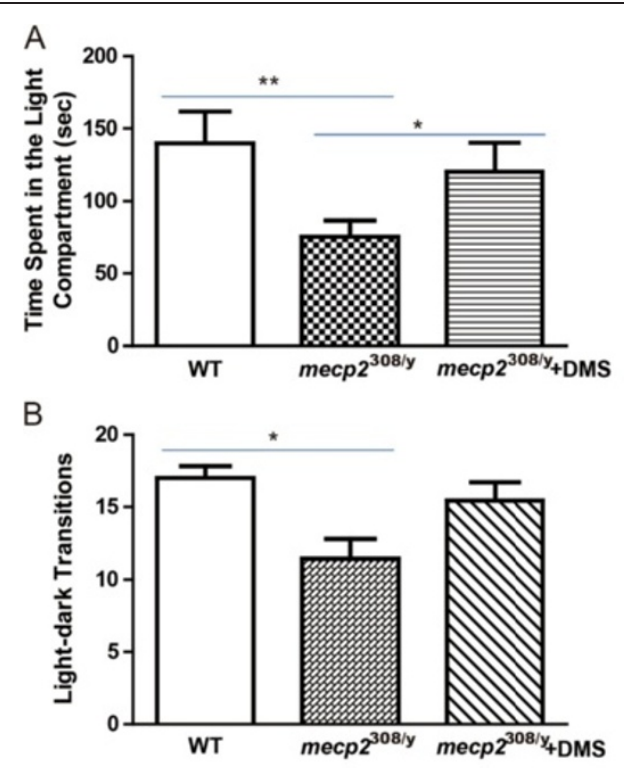

C

nestin-cre, mecp2 floxy

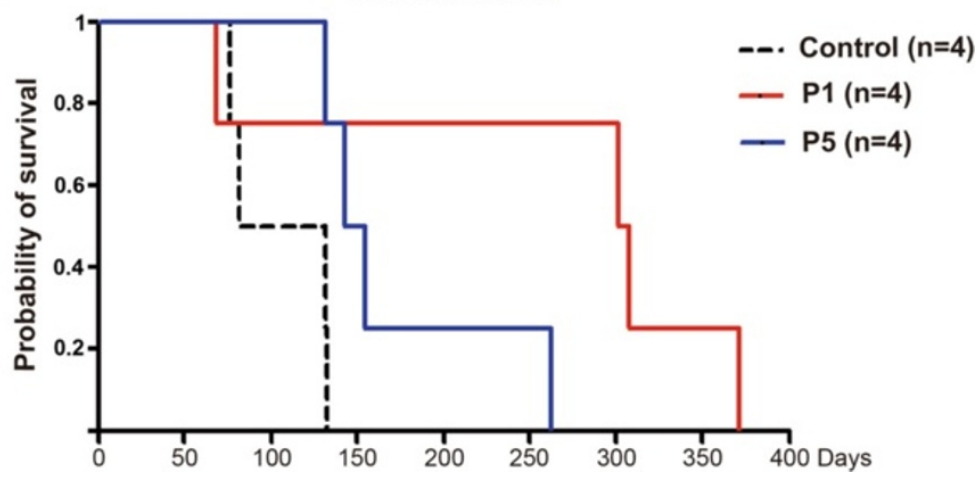

Figure 7 DMS alleviates anxiety-associated defects and extend life span in mouse model for Rett syndrome.

(A) Time spent in light compartment during light-dark transition tests. Values represent mean ( \pm ) SEM (Animal amounts, wt $n=8$, mecp $2^{308 / y} n=9$, mecp 2 $2^{308 / y}+$ DMS $n=9$; **: $P<0.005$, *: P<0.05, two-tailed student's t-test). (B) Times of light-dark transitions during test. Values represent mean ( \pm ) SEM (*: $\mathrm{P}<0.05$, two-tailed student's t-test). (C) Kaplan-Meier survive curve of nestin-cre, mecp $2^{\text {floxy }}$ mice with control, DMS with P1 and P5 treatments, respectively. ( $n=4$ for each condition).

Here we developed a novel rTMS paradigm, referred as DMS, to help the learned helpless animals recuperate from stress and depression. The short-term application of DMS to depressed mice not only improved their stress-related behavior but also amazingly reversed the pathophysiology of major depression, indicated by the alteration of molecular marker and neuronal plasticity. As the neurogenic hypothesis of depression gains momentum over the last decade, more and more evidence confirmed that the waning and waxing of neurogenesis in the hippocampus are important causal factors in the precipitation of and recovery from depression, respectively. Our study provided several lines of evidences to prove that DMS treatment increases adult neurogenesis and newborn neuron maturation in vivo. First, short-term DMS administration rapidly induces the proliferation of adult neural progenitors in the subgranular zone of DG. The enhanced neurogenesis by DMS treatment occurs in both adult and senescent animals. Second, the application of DMS to adult animals promotes the dendritic development new-born granule neurons, suggesting the elevated incorporation of new-born neurons into existing hippocampal circuitry might be achieved by magnetic stimulation. Finally, DMS treatment is shown to up-regulate the neural activity, which is beneficial to increase the production and release of neurogenic niche factors such as FGF1b. Thus, our results support the notion that DMS is an efficient therapeutic treatment for major depression disorders and the antidepressant effects of DMS may rely on the elevation of hippocampal adult neurogenesis and the modulation of hippocampal synaptic plasticity. 
Neural activity and experience, presumably acting on this local niche, regulate multiple stages of adult neurogenesis, from neural progenitor proliferation to newborn neuron maturation, synaptic integration and survival. Our study shows a new non-invasive way to enhance adult hippocampal neurogenesis probably by changing the network activity of hippocampus and inducing a beneficial neurogenic niche in the subgranular zone. Repetitive electrical stimulation in adult hippocampal slices induces NMDA receptor-dependent LTP and high frequency stimulation results in persistent increase in synaptic strength [39]. Studies using theta-burst stimulation protocols provide solid evidence linking human rTMS with LTP-like plasticity $[40,41]$. The DMS protocol we applied exerts high frequency oscillated stimulation in the brain of animal models and rescues the deficient LTP impaired by restraint stress in rats. On the other hand, LTP induction in the anesthetized rats increases neurogenesis in the dentate gyrus and FGFR activation by the neural cell adhesion molecule promotes neural progenitor proliferation by enhancing LTP $[42,43]$. Therefore, the study suggests that DMS administration may promote adult neurogenesis by inducing high frequency theta-burst stimulation and LTP in the hippocampus. Further study will be performed to explore the alteration of field potential by DMS treatment. Moreover, the finding that DMS alleviates stress response and prolongs the life expectancy of mouse model for Rett syndrome is particularly intriguing. Many trials have been carried out to examine whether drug delivery or genetic manipulation help to alleviate the impaired behavior and severe pathophysiology of Rett syndrome mouse model [44-46]. Although gene delivery of Mecp2 seems to completely rescue various symptoms in animal models of disease, the efficacy and safety issues of gene delivery remain controversial. Here our results suggest a modified DMS paradigm which may relieve the stressrelated symptoms, as well as improve the life quality of Rett syndrome patients. Further study of how DMS treatment may contribute the neural plasticity of the central nervous system under disorder conditions are very critical for deepen our understanding of therapeutic efforts towards the cure of neurodevelopmental disorders and neuropsychiatric disorders. In order to develop easier and more effective therapy for patients with neurodevelopmental disorders and neuropsychiatric disorders, we need to better understand how DMS treatment contributes to the neural plasticity of central nervous system under pathological conditions.

\section{Conclusion}

Taken together, we report that a new DMS paradigm rapidly induces adult hippocampal neurogenesis and promotes development of DG new-born neurons. Our study provides a new non-invasive way to enhance adult hippocampal neurogenesis within a short period of time and make it possible to study the role of adult hippocampal neurogenesis in a gain-of-function manner. More importantly, we provided evidences that this new DMS paradigm efficiently rescues behavioral phenotypes and gene expression profiles in learned helplessness mouse model, as well as restoring hippocampal LTP impaired by restraint stress in rats. These results support the notion that DMS is an efficient therapeutic treatment for major depression disorders and strongly suggest that the antidepressant effect of DMS on depression disorders may rely on the improvement of hippocampal adult neurogenesis and the correction of hippocampal synaptic plasticity.

\section{Methods}

\section{Animals}

Animals were group-housed with free access to water and food in the established animal houses, with a 12 hours light/dark cycle and a thermo-regulated environment. The use and care of animals complied with the guideline of the Biomedical Research Ethics Committee at the Shanghai Institutes for Biological Science, CAS. Adult (6-7 weeks old) male C57BL/6 mice (SLAC Laboratory Animal) were used in all experiments except electrophysiology trials which were performed on male Sprague Dawley rats (Animal House center, Kunming General Hospital, Kunming), weighing 220-250 g. mecp $2^{308 / y}$ mice (005439) and nestin-cre mice (003771) were purchased from Jackson lab; floxed mecp2 (011918) were purchased from MMRRC at UC Davis.

\section{DMS treatment}

For DMS treatment, mice with their cage were placed in DMS machine. Metal parts of cages were removed prior to DMS treatment. The applied program was described in Supplementary Figures. Briefly, twenty minutes successive trains of DMS were administered daily for different consecutive days depending on the purpose of experiments.14 days DMS were administered on retrovirus injected mice and 4 or 7 days DMS on Brdu labeled mice while 5 days DMS on acute induced depression mice. The control group conditions were identical to their DMS group but received sham stimulation. For electrophysiology experiments, control group received no treatment, Restraint stress (RS) group were restrained in the fixing cage 20 min each day for 7 days, RS + DMS group received 20 min DMS treatment in the fixing cage for 7 days. Electrophysiology studies were carried out $0.5 \mathrm{~h}$ later after the last restraint stress or DMS treatment.

\section{In vivo electrophysiology}

Experiments were carried out on rats anesthetized with pentobarbital (60 mg/kg, i.p.), and core temperature was 
maintained at $37 \pm 0.5^{\circ} \mathrm{C}$. Recordings of field excitatory postsynaptic potentials (EPSPs) were made from the CA1 stratum radiatum of the hippocampus in response to ipsilateral stimulation of the Schaffer collateral/commissural pathway using techniques described previously $[28,47]$. Recording and stimulating electrodes were made by gluing together a pair of twisted Teflon-coated $90 \%$ platinum and $10 \%$ iridium wires $(50 \mu \mathrm{m}$ inner diameter, $75 \mu \mathrm{m}$ outer diameter; World Precision Instruments, Sarasota, FL). The recording electrode was inserted $3.8 \mathrm{~mm}$ posterior to bregma and $2.8 \mathrm{~mm}$ right of the midline, and the stimulating electrode was inserted $4.8 \mathrm{~mm}$ posterior to bregma and $3.8 \mathrm{~mm}$ right of the midline. The optimal depth of the wire electrodes in the stratum radiatum of the CA1 area of the dorsal hippocampus was determined by electrophysiological criteria. Test EPSPs were evoked at a frequency of $0.033 \mathrm{~Hz}$ and at a stimulus intensity adjusted to give an EPSP amplitude $50 \%$ maximum response. The high frequency stimulation (HFS) protocol for inducing LTP consisted of 10 stimulus trains of 20 pulses at $200 \mathrm{~Hz}$, with $2 \mathrm{~s}$ intertrain intervals. EPSP amplitude was expressed as mean \pm S.E.M\% of the baseline EPSP amplitude recorded over a 40-min baseline period, and amplitudes in the last 10-min of recording were averaged in one animal and then across animals to give a value for the group.

\section{Preparation and stereotaxic injection of retrovirus}

We thank Drs. Hongjun Song (Johns Hopkins University School of Medicine) and Zhengang Yang (Institutes of Brain Science, Fudan University) for providing retroviral constructs and supernatant containing retrovirus. Cell debris were removed from supernatant through $0.22 \mu \mathrm{m}$ filter and the filtered supernatant was centrifuged at $65000 \mathrm{~g}$ at $4^{\circ} \mathrm{Cf}$ for $2 \mathrm{~h}$. Then supernatant was removed and $50 \mu \mathrm{l}$ PBS was used to re-suspend virus. We seed $293 \mathrm{~T}$ to determine the titer of virus. High titer virus of $10^{8} \mathrm{vg} / \mathrm{ml}$ was necessary to label new-born neurons in the adult DG.

For stereotaxic injection, it is critical to locate the exact hippocampus neurogenic area. The mouse was mounted onto the stereotaxic frame, orienting the head straight in terms of anterior-posterior axis and horizontally. Then we shaved a small area by a trimmer, cut the skin over the scull, cleaned up the blood in the wound.

The needle was firstly moved to the bregma and then moved posteriorly $2.0 \mathrm{~mm}$ and laterally $1.5 \mathrm{~mm}$ to the injection position. A small hole was made on the scull using an electric drill and the needle tip was moved down by $2.25 \mathrm{~mm}$ from the water level of the hole. $1 \mu \mathrm{l}$ retrovirus solution was injected to one side.

For both Brdu and retrovirus stereotaxic injection statistics, each group (control, program1 and program5) has 4-5 mice. Each mouse was collected 9-12 slices from dorsal to ventral region of DG. And 1 or 2 intact neurons were chosen from each slice for analysis. ImageJ software (http://rsbweb.nih.gov/ij/) was used to trace the dendrites of new-born neuron. Then the dendritic length and tips number were auto-analyzed.

\section{Bromodeoxyuridine injection}

Mice received four or seven days DMS treatment and Bronodeoxyuridine (Brdu) $(50 \mathrm{mg} / \mathrm{kg}$ in saline, every two hours for four times, Sigma, flu/Ald, B5002) was intraperitoneally (i.p.) injected into the control or DMS group mice 12 hours after the last DMS treatment. Mice were killed two hours later after the last i.p. injection.

\section{Tissue preparation, immunostaining and imaging}

Mice were transcardially perfused with $0.1 \mathrm{M}$ cold PBS followed by $4 \%$ paraformaldehyde (PFA) fixation. The brains were post-fixed for 12 hours in 4\% PFA and dehydrated in 30\% sugar solution for another 12 hours. Coronal sections of $40-\mu \mathrm{m}$ thickness were cut on the freezing microtome (LEICA CM1950) and stored in PBS.

For Brdu immunostaining, free-floating sections were incubated in $2 \mathrm{~N} \mathrm{HCl}$ for $15 \mathrm{~min}$ at 37 degree then neutralized in $0.1 \mathrm{M}$ boric acid solution $(\mathrm{pH} \mathrm{8.5)}$ for $10 \mathrm{~min}$ and washed by PBS for 3 times (each time for 5 minutes). Sections were incubated in mouse anti-Brdu antibody (1:1500; MAB3510) at 4 degree overnight and washed again then incubated in CF555 conjugated donkey antimouse secondary antibody (1:500; Biotum,20037) for 2.5 hours at room temperature.

For GFP immunostaining, free-floating sections were incubated in mouse anti-GFP antibody (1:1000;Santa Cruz, 9996) at 4 degree overnight and washed again then incubated in CF488 conjugated goat anti-mouse secondary antibody (1:500;Biotum,20010) for 2.5 hours (dark) at room temperature.

Immunostaining sections were photographed in Z-series stacks using a Nikon A1 confocal microscope. NIH Image J with NeuronJ plugin was used to count the Brdu-immunoreactive cell number in the dentate gyrus and to quantify the area of the dentate gyrus, also to analyze total dendritic length of $\mathrm{GFP}^{+}$new-born neurons.

\section{Statistical analysis}

The results were expressed as Mean \pm S.E.M. Statistical significance $(P<0.05)$ was assessed using the two-tailed Student's $t$-test.

\section{Learned helplessness paradigm}

Mice were placed into the inescapable shock chamber. 360 scrambled foot shocks $(0.6 \mathrm{~mA})$ with varying duration (1-3s) and interval-episodes (1-15s) were delivered over two consecutive days. Control group did not receive foot shocks but were placed to the shock chamber with equal time. On the first and second days, 8 hours 
after foot shocks, mice were administered DMS. Then mice were administered DMS for another 3 days.

\section{Forced swim test}

Mice were placed individually in the testing cylinder (33 cm high $\times 10 \mathrm{~cm}$ in diameter) containing water with $20 \mathrm{~cm}$ depth. The procedures were conducted as two minutes pretest followed by four minutes test and both sessions were videotaped. Observers scored the immobility (floating passively with slight movements) time in the test four minutes.

\section{Gamma irradiation}

Mice were anesthetized with $0.7 \%$ Pentobarbital sodium at $10 \mathrm{ul} / \mathrm{g}$, and exposed to cranial irradiation at a field of $3.5 \times 11 \mathrm{~mm}$ above the hippocampus but other body parts were protected with a $75 \mathrm{~mm}$ lead shield. Caesium-137 was used as radioactive source operated by MDS Nordion GC-3000Elan. The dose rate was approximately 4 Gy per min. The procedure lasted $5 \mathrm{~min}$ and $30 \mathrm{sec}$, delivering a total of 20 Gy in the center.

\section{Light-dark transition}

mecp $2^{308 / y}$ mutant mice were carried out light-dark transition test after daily DMS stimulation for 5 month at the age of 5-6 month. The chamber is divided into two unequal compartments by an opaque shelter with a hole (of height $4 \mathrm{~cm}$ ) at the floor level. The smaller compartment $(18 \mathrm{~cm} \times 27 \mathrm{~cm} \times 30 \mathrm{~cm})$ is painted black and covered by an opaque lid while the larger compartment $(27 \mathrm{~cm} \times 27 \mathrm{~cm} \times 30 \mathrm{~cm})$ is uncovered and is illuminated by ceiling room lights. Firstly, mice were gently placed into the dark compartment. Transition between the two compartments and time spent in the light compartment were automatically recorded by the Ethovision videotracking system in a $10 \mathrm{~min}$ session.

\section{Quantitative real time PCR}

Total RNA was collected from fresh hippocampus tissue for reverse transcription (Bio-Rad, 1708891). $2 \times$ SYBR Green Master Mix (TOYOBO, QPK201) and QIAGEN Rotor-Gene $\mathrm{Q}$ machine were used in real time PCR experiments. Mouse MKP-1 real time primer: forward: CGCTTCTCGGAAGGATATGCT; reverse: GTCAATA GCCTCGTTGAACCAG. Fgf1b primers were used as reported [23].

\section{Additional files}

Additional file 1: Figure S1. Illustration of DMS equipment and magnetic fields. (A) The positions of animal and DMS machine are showed. (B-C) Illustration of magnetic vector potential generated during Uniform and Linear phase by DMS equipment. (D-E) Illustration of Uniform and Linear magnetic fields generated by this equipment.
Additional file 2: Figure S2. The effect of DMS to adult hippocampal neurogenesis. A) Hippocampal DG Ki67 and BrdU immunostaining on mice with continuous DMS with P5 treatment for 7 days. Blue: DAPI; Green: Ki67; Red BrdU staining. (B) Quantification of (A). (C) Hippocampal DG Ki67 immunostaining on mice with continuous DMS with P5 treatment for 7 days. Blue: DAPI; Green: Ki67 staining. Lower right panel: Amplification of boxed area of (A) panel. (E) Hippocampal DG GFAP and BrdU immunostaining on mice with continuous DMS with P5 treatment for 7 days. Blue: DAPI; Green: GFAP; Red BrdU staining. (F) Quantification of (E). (G) Hippocampal DG GFAP immunostaining on mice with continuous DMS with P5 treatment for 7 days. Blue: DAPI; Green: GFAP staining. Lower right panel: Amplification of boxed area of $(\mathrm{G})$ panel. Scale bar $=100 \mu \mathrm{m}$.

Additional file 3: Figure S3. The effect of DMS to adult hippocampal neurogenesis (Continued). (A) Hippocampal DG Tbr2 and BrdU immunostaining on mice with continuous DMS with P5 treatment for 7 days. Blue: DAPI; Green: Tbr2; Red BrdU staining. (B) Quantification of (A). (C) Hippocampal DG Tbr2 immunostaining on mice with continuous DMS with P5 treatment for 7 days. Blue: DAPI; Green: Ki67 staining. Lower right panel: Amplification of boxed area of (C) panel. (E) Hippocampal DG DCX and BrdU immunostaining on mice with continuous DMS with P5 treatment for 7 days. Blue: DAPl; Green: DCX; Red BrdU staining. (F) Quantification of (E). (G) Hippocampal DG DCX immunostaining on mice with continuous DMS with P5 treatment for 7 days. Blue: DAPI; Green: DCX staining. Lower right panel: Amplification of boxed area of $(G)$ panel. Scale bar $=100 \mu \mathrm{m}$.

Additional file 4: Figure S4. The effect of DMS to development of mature hippocampal DG neurons. Example pictures of Golgi staining for hippocampal DG neuron in control (A) and DMS (B) condition. (C) Golgi staining for spines on hippocampal DG neurons. (D) Quantification of (C). (E) Immunohistochemistry for c-fos protein in laternal habenula region after DMS with P5 treatment for 4 days. (F) Quantification of (E).

Additional file 5: Figure S5. The effect of DMS on stress-induced depressive phenotypes in rodent models. (A) Outline of foot-shock-induced depressive behavioral paradigm and DMS treatment experiments. (B-E) Behavioral effects of learned helplessness mouse model with DMS treatment during various time points. Values represent mean $( \pm$ ) SEM (Animal amount: control $n=15$, Footshock $n=19$, Footshock with DMS $n=15$; **: $P<0.005$, two-tailed student's t-test.

Additional file 6: Figure S6. IR inhibits adult hippocampal neurogenesis in rodents. (A) Outline of gamma irradiation (IR) and BrdU injection experimentsHippocampal DG BrdU immunostaining on control mice (B), and with IR treatment (C). Red: BrdU; Blue: DAPI staining. (D) Quantification of (C,D). Values represent mean $( \pm)$ SEM $(n=12-16$ for each condition; *: $P<0.05$, two-tailed student's t-test). Scale bar $=100 \mu \mathrm{m}$.

\section{Competing interests}

The authors declare that they have no competing interests.

\section{Authors' contributions}

$\mathrm{LX}$ and ZQ conceived and supervised the project. $Y Z$ performed the majority of the work. RRM and MT performed electrophysiology work on rat. ZFC, MH, $Z R G, X L, D L D$, participated in early stage of this work including immunohistochemistry and daily DMS treatment for animals. JW designed the DMS machine. XX and WHZ and CYL participated in testing and data collection of DMS machine on animals. ZQ wrote the manuscript, with contributions from other authors. All authors read and approved the final manuscript.

\section{Acknowledgements}

We thank Dr. Bai Lu for his constructive comments for this work. This work was supported by the National Basic Research Program of China (2011CBA00400 to Z.Q., 2013 CB835103 and 2009CB941302 to L.X.), CAS Hundreds of Talents Program (Z.Q.), Strategic Priority Research Program of Chinese Academy of Sciences, Grant No.XDB02050400 (Z.Q.) and the National Natural Science Foundation of China (U1032605 to L.X, 31100786 to R. M.). 


\section{Author details}

'Institute of Neuroscience, Shanghai Institutes for Biological Sciences, Chinese Academy of Sciences, Shanghai, China. ${ }^{2}$ Key Laboratory of Animal Models and Human Disease Mechanisms, Laboratory of Learning and Memory, Kunming Institute of Zoology, Chinese Academy of Sciences, Kunming, Yunnan, China. ${ }^{3}$ School of Electrical and Automation Engineering, Tianjin University, Tianjin, China. ${ }^{4}$ Departments of Neonatology, Children's Hospital of Fudan University, Shanghai, China. ${ }^{5}$ Department of Child Healthcare, Children's Hospital of Fudan University, Shanghai, China.

Received: 5 September 2013 Accepted: 6 February 2014

Published: 11 February 2014

\section{References}

1. Merton PA, Morton HB: Stimulation of the cerebral cortex in the intact human subject. Nature 1980, 285:227-227.

2. Dayan E, Censor N, Buch ER, Sandrini M, Cohen LG: Noninvasive brain stimulation: from physiology to network dynamics and back. Nat Neurosci 2013, 16:838-844.

3. Hallett M: Transcranial magnetic stimulation: a primer. Neuron 2007, 55:187-199.

4. Belmaker RH, Agam G: Major depressive disorder. N Engl J Med 2008 358:55-68.

5. George MS, Taylor JJ, Short EB: The expanding evidence base for rTMS treatment of depression. Curr Opin Psychiatry 2013, 26:13-18.

6. Kammer T, Spitzer M: Brain stimulation in psychiatry: methods and magnets, patients and parameters. Curr Opin Psychiatry 2012, 25:535-541.

7. Arias-Carrión O: Basic mechanisms of rTMS: implications in Parkinson's disease. Int Arch Med 2008, 1:2.

8. Barker AT: An introduction to the basic principles of magnetic nerve stimulation. J Clin Neurophysiol 1991, 8:26-37.

9. Hoogendam JM, Ramakers GMJ, Di Lazzaro V: Physiology of repetitive transcranial magnetic stimulation of the human brain. Brain Stimul 2010, 3:95-118.

10. Chen R, Classen J, Gerloff C, Celnik P, Wassermann EM, Hallett M, Cohen LG: Depression of motor cortex excitability by low-frequency transcranial magnetic stimulation. Neurology 1997, 48:1398-1403.

11. Pasley BN, Allen EA, Freeman RD: State-dependent variability of neuronal responses to transcranial magnetic stimulation of the visual cortex. Neuron 2009, 62:291-303.

12. Perini F, Cattaneo L, Carrasco M, Schwarzbach JV: Occipital transcranial magnetic stimulation has an activity-dependent suppressive effect. J Neurosci 2012, 32:12361-12365.

13. Sahay A, Hen R: Adult hippocampal neurogenesis in depression. Nat Neurosci 2007, 10:1110-1115.

14. Malberg JE, Eisch AJ, Nestler EJ, Duman RS: Chronic antidepressant treatment increases neurogenesis in adult Rat hippocampus. J Neurosci 2000, 20:9104-9110

15. Van Praag H, Christie BR, Sejnowski TJ, Gage FH: Running enhances neurogenesis, learning, and long-term potentiation in mice. Proc Natl Acad Sci USA 1999, 96:13427-13431.

16. Czéh B, Michaelis T, Watanabe T, Frahm J, de Biurrun G, van Kampen M, Bartolomucci A, Fuchs E: Stress-induced changes in cerebral metabolites, hippocampal volume, and cell proliferation are prevented by antidepressant treatment with tianeptine. Proc Natl Acad Sci USA 2001, 98:12796-12801.

17. Hummel F, Celnik P, Giraux P, Floel A, Wu W-H, Gerloff C, Cohen LG: Effects of non-invasive cortical stimulation on skilled motor function in chronic stroke. Brain 2005, 128(Pt 3):490-499.

18. Miniussi C, Cappa SF, Cohen LG, Floel A, Fregni F, Nitsche MA, Oliveri M, Pascual-Leone A, Paulus W, Priori A, Walsh V: Efficacy of repetitive transcranial magnetic stimulation/transcranial direct current stimulation in cognitive neurorehabilitation. Brain Stimul 2008, 1:326-336.

19. Kuhn H, Dickinson-Anson H, Gage F: Neurogenesis in the dentate gyrus of the adult rat: age-related decrease of neuronal progenitor proliferation. J Neurosci 1996, 16:2027-2033.

20. Zhao C, Deng W, Gage FH: Mechanisms and functional implications of adult neurogenesis. Cell 2008, 132:645-660.

21. Ming $\mathrm{G}$, Song $\mathrm{H}$ : Adult neurogenesis in the mammalian central nervous system. Annu Rev Neurosci 2005, 28:223-250.
22. Van Praag H, Schinder AF, Christie BR, Toni N, Palmer TD, Gage FH: Functional neurogenesis in the adult hippocampus. Nature 2002, 415:1030-1034.

23. Ma DK, Jang M-H, Guo JU, Kitabatake Y, Chang M-L, Pow-Anpongkul N, Flavell RA, Lu B, Ming G-L, Song H: Neuronal activity-induced Gadd45b promotes epigenetic DNA demethylation and adult neurogenesis. Science (New York, NY) 2009, 323:1074-1077.

24. Ernst C, Olson AK, Pinel JPJ, Lam RW, Christie BR: Antidepressant effects of exercise: evidence for an adult-neurogenesis hypothesis? J Psychiatry Neurosci 2006, 31:84-92.

25. Feng S, Shi T, Fan-Yang, Wang W, Chen Y, Tan Q: Long-lasting effects of chronic rTMS to treat chronic rodent model of depression. Behav Brain Res 2012, 232:245-251.

26. Chourbaji S, Zacher C, Sanchis-Segura C, Dormann C, Vollmayr B, Gass P: Learned helplessness: validity and reliability of depressive-like states in mice. Brain Res Brain Res Protoc 2005, 16:70-78.

27. Duric V, Banasr M, Licznerski $P$, Schmidt HD, Stockmeier CA, Simen AA, Newton SS, Duman RS: A negative regulator of MAP kinase causes depressive behavior. Nat Med 2010, 16:1328-1332.

28. Dong Z, Han H, Cao J, Xu L: Opioid withdrawal for 4 days prevents synaptic depression induced by low dose of morphine or naloxone in rat hippocampal CA1 area in vivo. Hippocampus 2010, 20:335-343.

29. Foy MR, Stanton ME, Levine S, Thompson RF: Behavioral stress impairs long-term potentiation in rodent hippocampus. Behav Neural Biol 1987, 48:138-149.

30. Xu L, Anwyl R, Rowan MJ: Behavioural stress facilitates the induction of long-term depression in the hippocampus. Nature 1997, 387:497-500.

31. Xu L, Anwyl R, Rowan MJ: Spatial exploration induces a persistent reversal of long-term potentiation in rat hippocampus. Nature 1998, 394:891-894.

32. Kim JJ, Diamond DM: The stressed hippocampus, synaptic plasticity and lost memories. Nat Rev Neurosci 2002, 3:453-462.

33. Amir RE, Van den Veyver IB, Wan M, Tran CQ, Francke U, Zoghbi HY: Rett syndrome is caused by mutations in X-linked MECP2, encoding methyl-CpG-binding protein 2. Nat Genet 1999, 23:185-188.

34. Chahrour M, Zoghbi HY: The story of Rett syndrome: from clinic to neurobiology. Neuron 2007, 56:422-437.

35. Guy J, Cheval H, Selfridge J, Bird A: The role of MeCP2 in the brain. Annu Rev Cell Dev Biol 2011, 27:631-652.

36. Shahbazian M, Young J, Yuva-Paylor L, Spencer C, Antalffy B, Noebels J, Armstrong D, Paylor R, Zoghbi H: Mice with truncated MeCP2 recapitulate many Rett syndrome features and display hyperacetylation of histone H3. Neuron 2002, 35:243-254.

37. Guy J, Hendrich B, Holmes M, Martin JE, Bird A: A mouse Mecp2-null mutation causes neurological symptoms that mimic Rett syndrome. Nat Genet 2001, 27:322-326.

38. Chen RZ, Akbarian $S$, Tudor $M$, Jaenisch R: Deficiency of methyl-CpG binding protein-2 in CNS neurons results in a Rett-like phenotype in mice. Nat Genet 2001, 27:327-331.

39. Dudek SM, Bear MF: Bidirectional long-term modification of synaptic effectiveness in the adult and immature hippocampus. J Neurosci 1993, 13:2910-2918

40. Huang Y-Z, Edwards MJ, Rounis E, Bhatia KP, Rothwell JC: Theta burst stimulation of the human motor cortex. Neuron 2005, 45:201-206.

41. Di Lazzaro V, Pilato F, Dileone M, Profice P, Oliviero A, Mazzone P, Insola A, Ranieri F, Tonali PA, Rothwell JC: Low-frequency repetitive transcranial magnetic stimulation suppresses specific excitatory circuits in the human motor cortex. J Physiol 2008, 586(Pt 18):4481-4487.

42. Bruel-Jungerman E, Davis S, Rampon C, Laroche S: Long-term potentiation enhances neurogenesis in the adult dentate gyrus. J Neurosci 2006, 26:5888-5893.

43. Dallérac $G$, Zerwas M, Novikova T, Callu D, Leblanc-Veyrac P, Bock E, Berezin $V$, Rampon $C$, Doyère $V$ : The neural cell adhesion molecule-derived peptide FGL facilitates long-term plasticity in the dentate gyrus in vivo. Learn Mem 2011, 18:306-313.

44. Guy J, Gan J, Selfridge J, Cobb S, Bird A: Reversal of neurological defects in a mouse model of Rett syndrome. Science (New York, NY) 2007, 315:1143-1147.

45. Tropea D, Giacometti E, Wilson NR, Beard C, McCurry C, Fu DD, Flannery R, Jaenisch R, Sur M: Partial reversal of Rett Syndrome-like symptoms in MeCP2 mutant mice. Proc Natl Acad Sci USA 2009, 106:2029-2034. 
46. Giacometti E, Luikenhuis S, Beard C, Jaenisch R: Partial rescue of MeCP2 deficiency by postnatal activation of MeCP2. Proc Natl Acad Sci USA 2007, 104:1931-1936.

47. Yang Y, Zheng X, Wang Y, Cao J, Dong Z, Cai J, Sui N, Xu L: Stress enables synaptic depression in CA1 synapses by acute and chronic morphine: possible mechanisms for corticosterone on opiate addiction. J Neurosci 2004, 24:2412-2420.

doi:10.1186/1756-6606-7-11

Cite this article as: Zhang et al:: Deep-brain magnetic stimulation

promotes adult hippocampal neurogenesis and alleviates stress-related

behaviors in mouse models for neuropsychiatric disorders. Molecular

Brain 2014 7:11.

\section{Submit your next manuscript to BioMed Central and take full advantage of:}

- Convenient online submission

- Thorough peer review

- No space constraints or color figure charges

- Immediate publication on acceptance

- Inclusion in PubMed, CAS, Scopus and Google Scholar

- Research which is freely available for redistribution 